\title{
Study of human acellular amniotic membrane loading bone marrow mesenchymal stem cells in repair of articular cartilage defect in rabbits
}

\author{
P.-F. Liu, L. Guo, D.-W. Zhao, Z.-J. Zhang, K. Kang, R.-P. Zhu and \\ X.-L. Yuan \\ Department of Orthopaedics, Affiliated Zhongshan Hospital of Dalian University, \\ Dalian, China \\ Corresponding author: L. Guo \\ E-mail: linguodoc@163.com
}

Genet. Mol. Res. 13 (3): 7992-8001 (2014)

Received August 27, 2013

Accepted March 11, 2014

Published September 29, 2014

DOI http://dx.doi.org/10.4238/2014.September.29.12

\begin{abstract}
The aim of this study was to investigate the repair effect of human acellular amniotic membrane (HAAM) loading bone marrow mesenchymal stem cells (BMSCs) on articular cartilage defect in rabbits. Rabbit BMSCs were isolated and cultured, and they were then inoculated on HAAM to prepare the complex of HAAM and BMSCs. Twenty-four rabbits were randomly divided into groups A and $\mathrm{B}$, with 12 animals in each group. The left and right sides were used as the experimental and control sides, respectively. The models of bilateral articular cartilage defect were established. The defect areas on the experimental side in groups A and B were implanted with the complex of HAAM and BMSCs and HAAM alone, respectively. The control sides of the two groups were not implanted with any material. In the 8th and 12th week after surgery, gross observation, histological examination and cartilage defect scoring were performed. In the 8th and 12 th postoperative week, gross observation and histological observation showed that dense cartilage-like cells appeared in group A but not in group B, indicating preferable cartilage repair. The cartilage defect score
\end{abstract}


on the experimental side in group A was $5.31 \pm 0.68$ in the 8 th week and $3.23 \pm 0.52$ in the 12th week, and that in group A was significantly lower than in group $\mathrm{B}(\mathrm{P}<0.05)$. HAAM loading BMSCs has a good repair effect on articular cartilage defect under an in vitro environment.

Key words: Human acellular amniotic membrane; Cartilage defect; Bone marrow mesenchymal stem cells; Cartilage repair

\section{INTRODUCTION}

The self-repair ability of articular cartilage is extremely limited. The newly formed repair tissue is fibrous tissue, of which the physicochemical properties are greatly different from normal cartilage. Traditional repair measures cannot treat a cartilage defect with a diameter of more than $4 \mathrm{~mm}$. Even if the repair is implemented, the mechanical performance of repair tissue is poor, with a poor treatment outcome. With the development of bionics and tissue engineering, more and more investigators have prepared cell scaffolds and simulated the microenvironment for cartilage cell growth. The target cells are amplified in vitro and then combined with cell scaffolds. The complex is implanted in the cartilage defect to construct the tissue-engineered cartilage. All kinds of trauma and cartilage damage due to arthritis are accompanied by subchondral bone impairment. It is reported that the subchondral bone nourishes and supports the cartilage, provides a biomechanical environment for cartilage growth and differentiation (Frosch et al., 2006), and excretes the metabolic waste (Martin et al., 2007). Bone marrow mesenchymal stem cells (BMSCs) can be transformed into the cartilage and bone, promoting new tissue growth and integration with surrounding tissue (Swieszkowski et al., 2007). As immunoregulatory cells, BMSCs have an immunosuppressive effect, which will not disappear with differentiation (Niemeyer et al., 2007). Researchers have shown that after implantation into the articular cartilage defect, BMSCs can directly differentiate into cartilage cells in a cartilage microenvironment to form cartilage matrix and construct cartilage tissue. Wakitani et al. (2002) used a complex of autologous BMSCs and type I collagen gel to repair a full-thickness cartilage defect in rabbit knee and found that the cartilage and subchondral bone healed within 24 weeks after surgery. Haleem et al. (2010) also studied the repair of articular cartilage defect in femoral condyle using BMSCs combined with fibrin glue containing platelets. These studies have provided a theoretical basis for using BMSCs as seed cells in the present article. Human amniotic membrane (HAM) is an important cell scaffold material (Wakitani et al., 1994; Zhang and Chan, 2010). As pointed out by DíazPrado et al. (2010), HAM is a good cell loading material for repair of cartilage defect. In the present study, human acellular amniotic membrane (HAAM) was used to load BMSCs. After in vitro co-culture, the complex of HAAM and BMSCs was implanted in animals. The role of HAAM loading BMSCs in the repair of cartilage defect was investigated. The objective was to provide a foundation for applying HAAM loading BMSCs to articular cartilage defect repair.

\section{MATERIAL AND METHODS}

\section{Preparation of BMSCs}

Rabbit BMSCs were isolated and cultured using the whole bone marrow culture method 
(Wakitani et al., 1994). The third-generation cells with uniform morphology and without aging were collected for further experiments. This study was carried out in strict accordance with the recommendations in the Guide for the Care and Use of Laboratory Animals of the National Institutes of Health. The animal use protocol had been reviewed and approved by the Institutional Animal Care and Use Committee (IACUC) of Affiliated Zhongshan Hospital of Dalian University.

\section{Preparation of HAAM}

HAM was taken from the placental tissue of healthy parturient with cesarean section in our hospital. The individuals positive for HIV, HBV, HCV, or syphilis were excluded. After removing the blood clots on placental tissue using sterile normal saline, HAM was separated from the chorion by blunt dissection and was then marked on the epithelial surface. After wiping the amniotic mucus using gauze soaked with sterile normal saline, HAM was repeatedly washed with sterile normal saline containing gentamicin $(1000 \mathrm{U} / \mathrm{mL})$, penicillin $(100$ $\mathrm{U} / \mathrm{mL})$, streptomycin $(100 \mu \mathrm{g} / \mathrm{mL})$, and amphotericin $(2.5 \mu \mathrm{g} / \mathrm{mL})$, followed by immersion for $30 \mathrm{~min}$. After washing with sterile normal saline, it was digested with $0.25 \%$ trypsin for $30 \mathrm{~min}$. The residual amniotic epithelial cells were gently removed using a blunt instrument, and HAAM was obtained. The microscopy and hematoxylin-eosin (HE) staining of HAAM were performed. HAAM $(1 \times 1 \mathrm{~cm})$ was tiled on the sterile gauze, with epithelial surface up. Informed consent for this experiment was obtained from the parturient women.

\section{Inoculation of BMSCs}

The third-generation BMSCs (subconfluent state) were washed with PBS twice, and then inoculated onto HAAM $\left(1.63 \times 10^{5} \mathrm{cells} / \mathrm{cm}^{2}\right)$. The complex of HAAM and BMSCs was placed on a 24 -well plate, and $1 \mathrm{~mL}$ complete medium containing $10 \%$ fetal bovine serum was then added to each well. After 2 days of culture, the medium was discarded, and the non-adherent cells were flushed away with PBS. The medium was changed every 2-3 days. After 7-8 days of culture, the cells reached a subconfluent state. The fluorescence microscopy, transmission electron microscopy (TEM) and HE staining were conducted on the complex.

\section{Treatment of experimental animals}

A total of 24 New Zealand rabbits (ordinary grade, 4 months old, $4 \pm 0.8 \mathrm{~kg}$, regardless of gender, provided by Experimental Animal Center of Dalian University) were randomly divided into groups A and B, 12 animals in each group. The left and right sides were used as the experimental and control sides, respectively. After revealing the non-weight-bearing area of bilateral femoral condyles of knee, a 4-mm drill bit was used to drill into this area, until blood oozing and appearance of slight resistance. The pore was about $3 \mathrm{~mm}$ deep, and reached the subchondral bone. The articular cartilage defect model was thereby established. In group A, the defect area in experimental side was implanted with the complex of HAAM and BMSCs. In group B, the experimental side was implanted with only HAAM. In the defect area of the control side in the two groups, no any material was implanted. Finally, the incision was sutured.

After surgery, the rabbits were treated by intramuscular injection with penicillin $(80,000 \mathrm{U} / \mathrm{rabbit})$, and then reared in single cage and fed with routine feedstuff ad libitum. In the 8 th and 12th postoperative week, 6 rabbits in each group were euthanized. According to 
the original surgical approach, the skin and subcutaneous tissue were incised. The new tissue in the bilateral defect area was taken, and the gross observation and histological examination were performed. The postoperative histological specimens were scored for cartilage defect according to the modified Wakitani scoring method (Wakitani et al., 2002).

\section{Statistical analysis}

Data are reported as means \pm SD. Statistical analysis was performed using the SPSS 10.0 statistical software. A $t$-test was used to analyze the differences between the two groups, and $\mathrm{P}<0.05$ was considered to be statistically significant.

\section{RESULTS}

\section{Microscopic observation of BMSCs}

The medium was first changed after culture of primary BMSCs for $24 \mathrm{~h}$. The vast majority of cells were adherent, with round morphology. Scattered fusiform or triangular cells were visible and showed strong refractivity (Figure 1A). After $48 \mathrm{~h}$, the number of fusiform and triangular cells with strong refractivity increased, with prominence at both ends of cell (Figure 1B). At $72 \mathrm{~h}$, there were mainly fusiform cells, and spirally arranged colonies appeared. The suspended cells were gradually removed with more frequent medium change (Figure 1C). On the 8th day, the cells reached a subconfluent state, with unclear cell outline. The cells almost covered the bottom of the bottle, with crowded growth (Figure 1D).

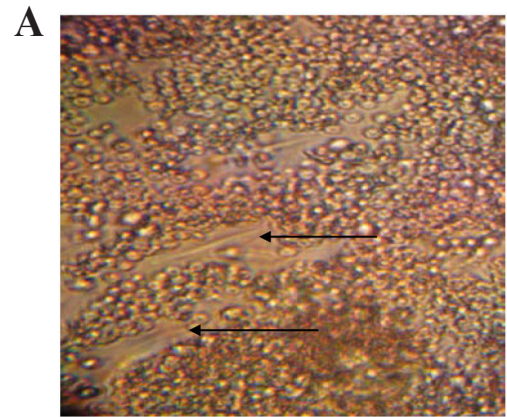

C

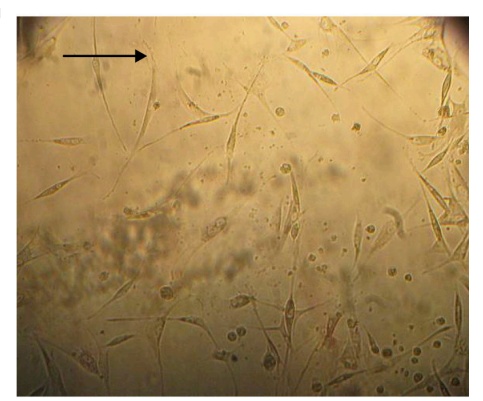

B

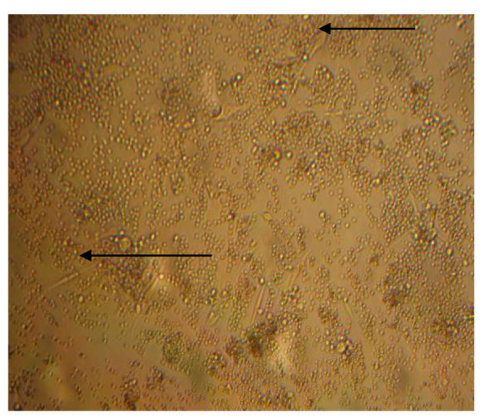

D

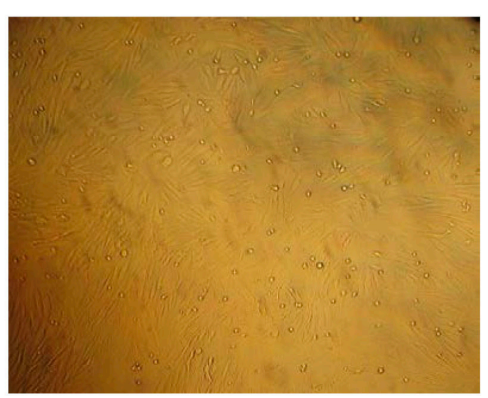

Figure 1. Microscopic observation of BMSCs. A. Microscopy of primary BMSCs (24 h). B. Microscopy of primary BMSCs (48 h). C. Microscopy of primary BMSCs (72 h). D. Microscopy of primary BMSCs (8 days). 
The passaged cells were completely adherent within $3 \mathrm{~h}$, with uniform distribution and rapid growth, but no spirally arranged colony was visible. On the 3rd to 5th day, the cells were confluent and were fused into scaly morphology. The cell arrangement was more orderly than primary cells, with a few impure cells. Compared with primary cells, a few of the fifthgeneration cells had abnormal morphology, hypertrophic cell body, increased cytoplasmic granules, and decreased refractivity, indicating the cell aging.

\section{Observation of complex of HAAM and BMSCs}

After 10 days of culture, TEM of the complex of HAAM and BMSCs showed that the cell nucleus was large and oval. The cells were spindle-shaped with prominence at both ends. For some cells, the prominence was long, with a large nucleus-cytoplasm ratio. These characteristics were in line with the ultrastructure features of BMSCs. The cells were tightly adherent to HAAM (Figure 2). HE staining showed that the BMSCs were attached to the scaffolds (Figure 3).

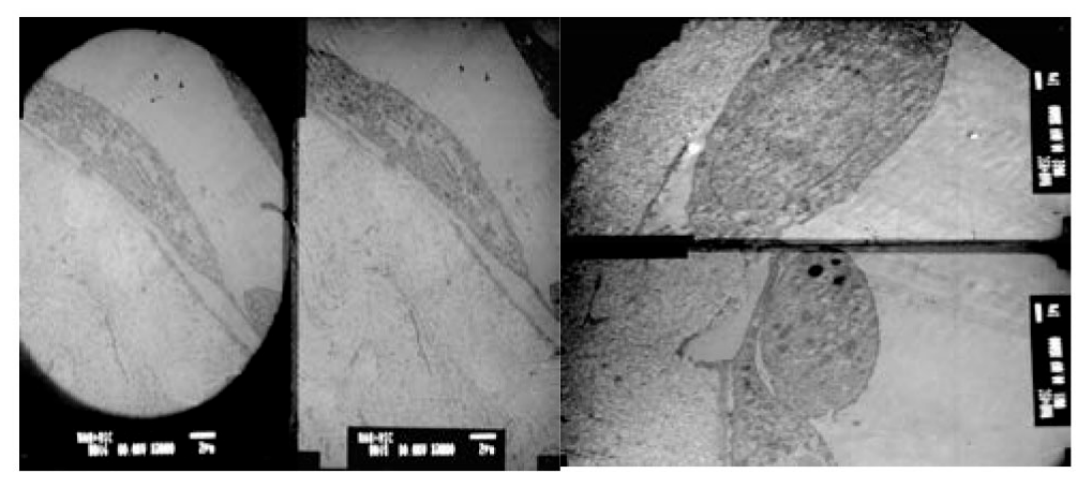

Figure 2. TEM on complex of HAAM and BMSCs.

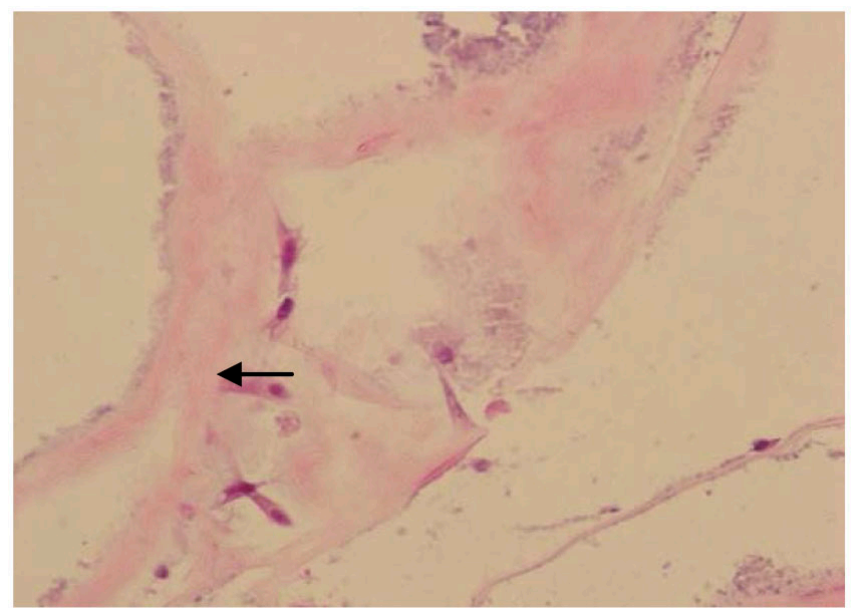

Figure 3. HE staining on complex of HAAM and BMSCs. 


\section{Gross observation}

After surgery, the rabbit knee joint had normal activities, with grade I incision healing. There was no infection or loose body formation in the joint, with no osteophyte formation in the defect area. On the 8th postoperative week, there was new white tissue in the defect area on the experimental side in group A, with a smooth surface. The formation of cartilage-like tissue was visible. The repair surface was lower than in surrounding tissue. In group B, there was also new white tissue in the defect area on the experimental side, with hard texture and less smooth surface. The repair surface was lower than in surrounding tissue, with no formation of cartilage-like tissue.

In the 12th postoperative week, there was smooth new tissue in the defect area on the experimental side in group A, with good integration with surrounding normal cartilage. The color of new tissue was similar with surrounding cartilage. The range of cartilage-like tissue increased, and the texture was soft but harder than granulation tissue (Figure 4A). In group B, the new milky white tissue on the experimental side was fiber-like tissue with smooth surface and hard texture. On the control side in the two groups, in the 8th and 12th weeks, the new tissue was milky white, and the texture was hard but relatively softer than bone. In the 12th week, the repair surface was even with surrounding tissue (Figure 4B).

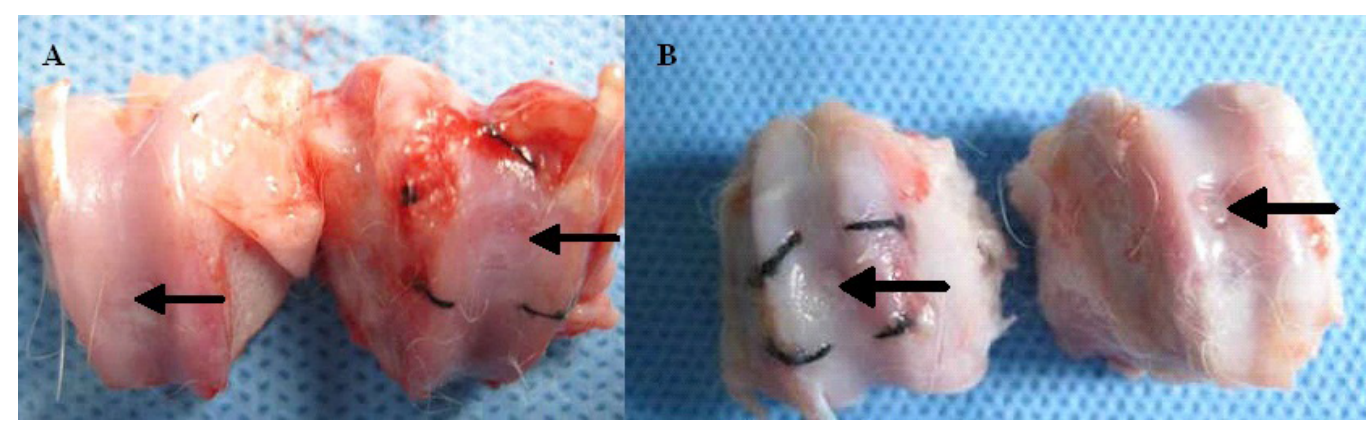

Figure 4. Gross observation results. A. Gross observation of articular cartilage in group A at the 12th postoperative week. (left, control side; right, experimental side). B. Gross observation of articular cartilage in group B at the 12th postoperative week. (left, experimental side; right, control side).

\section{Histological examination}

In the 8th postoperative week, there were mainly circular hyaline cartilage-like cells on the experimental side in group A, with formation of cartilage lacunae. The cartilage-like cells were located in cartilage lacunae, and appeared progressively mature with depth. The cells were scattered, and type II collagen staining was positive (Figure 5A). In group B, there was mainly fibrous-like tissue on the experimental side and control side, which contained a small amount of inflammatory cells, without formation of cartilage-like cells.

In the 12th week, on the experimental side of group A, there were mainly hyaline cartilage-like cells in cartilage lacunae, with increased number and larger body. There was much cartilage-like matrix, with normal coloring (Figure 5B). In group B, there were scattered cartilage-like cells in experimental side. On the control side of the two groups, no cartilagelike cells or tissue was visible (Figure 5C). 


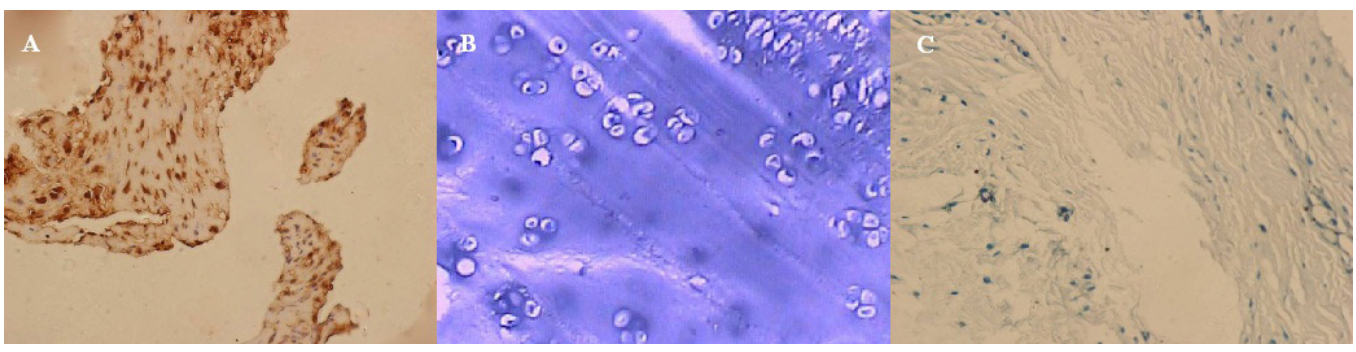

Figure 5. Histological examination results. A. Type II collagen staining of new tissue in experimental side of group A. B. Toluidine blue staining of new tissue in experimental side of group A. C. Toluidine blue staining of new tissue in control side of the two groups.

\section{Cartilage defect scoring}

Cartilage defect scores are shown in Tables 1 and 2. In the 8th postoperative week, in group $\mathrm{A}$, the cartilage defect score of the experimental side was significantly lower than for the control side $(\mathrm{P}<0.05)$. In group $\mathrm{B}$, the difference between the two sides was not statistically significant. In the 12th week, the score of the experimental side in the two groups was significantly lower than for the control side $(\mathrm{P}<0.05)$, and the score of the experimental side in group A was significantly lower than in group $\mathrm{B}(\mathrm{P}<0.05)$.

Table 1. Patient characteristics and deletion mutation.

\begin{tabular}{lcc}
\hline & HBeAg positive & HBeAg negative \\
\hline Case & 7 & 14 \\
Age (years; means \pm SD) & $37.4 \pm 12.3$ & $35.7 \pm 16.5$ \\
Infection history & $8.3 \pm 11.4$ & $9.6 \pm 15.4$ \\
Family history & 4 & 7 \\
Sequencing quantity & 27 & 47 \\
Deletion mutation & 20 & 35 \\
Normal & 7 & 12 \\
\hline
\end{tabular}

Table 2. Cartilage defect scoring in group B (means $\pm \mathrm{SD})$.

\begin{tabular}{lcr}
\hline Side & 8th postoperative week & 12th postoperative week \\
\hline Experimental & $8.37 \pm 0.59$ & $7.12 \pm 0.62$ \\
Control & $8.41 \pm 0.74$ & $8.26 \pm 0.69$ \\
\hline
\end{tabular}

\section{DISCUSSION}

In the cartilage repair-related area, bionics research has attracted more and more attention. Many investigators combine tissue engineering with biomechanics and simulate the microenvironment and mechanical stimulation conditions for articular cartilage growth, to establish tissue-engineered cartilage that is highly similar to normal cartilage. The main components of tissue-engineered cartilage are seed cells, scaffolds and growth factors. 
Seed cells are the most basic element of tissue engineering (Pittenger, 2008) and make up the main content for repair of articular cartilage defect. At present, seed cells mainly include BMSCs and cartilage cells. The cartilage cells have disadvantage of limited source, large allograft rejection, and phenotype loss with in vitro culture. However, BMSCs have the following advantages: 1) convenience in drawing material and adequate cell sources; 2) high in vitro proliferative capacity; and 3) BMSCs being immunoregulatory cells and able to inhibit the growth of B cells (Le Blanc et al., 2003), T cells (Ryan et al., 2007) and antigen-presenting cells and to naturally kill cells. These functions do not disappear after differentiation into other tissues (Le Blanc et al., 2003). In addition, allograft immune rejection of BMSCs is low. BMSCs were therefore selected for repair of cartilage defect in this study.

At present, BMSCs are mainly cultured using the whole bone marrow culture method (adherent screening method) and density gradient centrifugation method. The former was adopted in the present experiments. The main reasons were as follows. 1) The proliferative capacity of cells obtained using the whole bone marrow culture method is higher than when using the density gradient centrifugation method. This method can retain certain growth factors and adhesion-promoting material in bone marrow, and can produce fibroblast colony-forming units. 2) The separation liquid in density gradient centrifugation method is harmful to BMSCs. 3) The whole bone marrow culture method has the advantages of simple operation, short time, and low oxicity incidence. Using this method, the obtained third-generation BMSCs have high purity, and can be used as seed cells for tissue engineering.

There is no uniform standard for stem cell identification. In 2006, the International Mesenchymal and Tissue Stem Cell Committee issued 3 minimum identification standards (Dominici et al., 2006) for human MSCs for experimental study, which are as follows: 1) MSCs have adhesion ability to plastic substrate; 2) MSCs are positive for CD44 and CD90 but negative for CD45, CD34 and CD14; and 3) after in vitro induction, MSCs can be transformed into osteoblasts, adipocytes and cartilage cells for differentiation. Among these 3 criteria, the second has no universality, and the biological characteristics described in the first and third standard are also applicable to MSCs from animals. In this research, MSCs were mainly identified by morphology, surface markers, and in vivo induced differentiation into cartilage. As the cell adhesion is the main factor for separating BMSCs from other cells, the impure cells in the culture medium can be removed by repeated medium changes and passage. During cell passage, the time of trypsin digestion should be strictly controlled. In this study, the trypsin digestion was terminated within $30 \mathrm{~s}$ to $1 \mathrm{~min}$. Otherwise, the cells do not fully fall off the wall or are damaged, leading to significant decline in proliferative capacity.

HAM is a kind of natural translucent biological membrane with the following characteristics. 1) HAM contains ingredients promoting cell proliferation and differentiation, with more adhesion-promoting substances (e.g., collagen and laminin) (Dravida et al., 2008). 2) HAM has anti-inflammatory, antimicrobial, antifibrotic, and anti-scar effect, with appropriate mechanical properties and low immunity. It can express several growth factors (e.g., TGF) and provide abundant nutrients for cell proliferation and differentiation. In addition, HAM itself has anti-angiogenic and anti-inflammatory effects. It has been widely used in ophthalmology, cosmetology and repair of burn wounds for a long term (Rahman et al., 2009). 3) HAM can promote the transformation of BMSCs into cartilage (Tan et al., 2011). It can be concluded that HAM is an important cell scaffold material (Niknejad et al., 2008; Zhang and Chan, 2010). This provided a basis for using HAM as cell scaffolds in our experiments. 
In vitro culture of BMSCs is conducted in a simulated microenvironment for cartilage formation. The specific mechanism for transformation of BMSCs into cartilage is not clear. No kind of in vitro culture environment can substitute for the in vivo microenvironment for cartilage growth. Many researchers use in vivo cartilage environment to promote the chondrification of BMSCs, for repairing the cartilage defect. BMSCs can be chondrified in the local environment of the cartilage defect area. In the surrounding environment, the growth of some cells can affect that of other cells (Ahn et al., 2006). The cartilage cells can secrete various growth factors such as insulin-like growth factor and transforming growth factor (Hwang et al., 2007), which can promote the chondrification of BMSCs. A number of studies have indicated that the in vivo environment in the cartilage defect can induce the transformation of BMSCs into cartilage for defect repair.

The results of this study showed that in group B, the repair effect on the experimental side was better than on the control side. This indicated that there were some in vivo BMSCs participating in the repair. The repair effect on the experimental side in group A was better than in group B, suggesting that in vitro-amplified BMSCs had a dominant effect on cartilage defect repair. However, this needs to be further investigated. In this study, HAAM was used as a carrier of BMSCs. Removal of the inherent epithelial cells in HAM can reduce the immunogenicity of the amniotic membrane, thus preventing the intercellular rejection. The thirdgeneration BMSCs were inoculated on the basement surface of amniotic membrane. After 6 to 7 days of growth, the complex of HAAM and BMSCs was implanted in the cartilage defect area in rabbits. The results showed that there was cartilage-like tissue formation in the defect area, and that the cartilage defect was well repaired. It is confirmed that HAAM can be used as a kind of carrier for the repair of cartilage defect. However, the biomechanical adaptability and long-term structure stability of new cartilage tissue still need to be further studied.

\section{Conflicts of interest}

The authors declare no conflict of interest.

\section{REFERENCES}

Ahn SE, Kim S, Park KH, Moon SH, et al. (2006). Primary bone-derived cells induce osteogenic differentiation without exogenous factors in human embryonic stem cells. Biochem. Biophys. Res. Commun. 340: 403-408.

Díaz-Prado S, Rendal-Vázquez ME, Muiños-López E, Hermida-Gómez T, et al. (2010). Potential use of the human amniotic membrane as a scaffold in human articular cartilage repair. Cell Tissue Bank. 11: 183-195.

Dominici M, Le Blanc K, Mueller I, Slaper-Cortenbach I, et al. (2006). Minimal criteria for defining multipotent mesenchymal stromal cells. The International Society for Cellular Therapy position statement. Cytotherapy 8: 315-317.

Dravida S, Gaddipati S, Griffith M, Merrett K, et al. (2008). A biomimetic scaffold for culturing limbal stem cells: a promising alternative for clinical transplantation. J. Tissue Eng. Regen. Med. 2: 263-271.

Frosch KH, Drengk A, Krause P, Viereck V, et al. (2006). Stem cell-coated titanium implants for the partial joint resurfacing of the knee. Biomaterials 27: 2542-2549.

Haleem AM, Singergy AA, Sabry D, Atta HM, et al. (2010). The clinical use of human culture-expanded autologous bone marrow mesenchymal stem cells transplanted on platelet-rich fibrin glue in the treatment of articular cartilage defects: A pilot study and preliminary results. Cartilage 1: 253-261.

Hwang NS, Varghese S, Puleo C, Zhang Z, et al. (2007). Morphogenetic signals from chondrocytes promote chondrogenic and osteogenic differentiation of mesenchymal stem cells. J. Cell Physiol. 212: 281-284.

Le Blanc K, Tammik C, Rosendahl K, Zetterberg E, et al. (2003). HLA expression and immunologic properties of differentiated and undifferentiated mesenchymal stem cells. Exp. Hematol. 31: 890-896.

Martin I, Miot S, Barbero A, Jakob M, et al. (2007). Osteochondral tissue engineering. J. Biomech. 40: 750-765. 
Niemeyer P, Kornacker M, Mehlhorn A, Seckinger A, et al. (2007). Comparison of immunological properties of bone marrow stromal cells and adipose tissue-derived stem cells before and after osteogenic differentiation in vitro. Tissue Eng. 13: 111-121.

Niknejad H, Peirovi H, Jorjani M, Ahmadiani A, et al. (2008). Properties of the amniotic membrane for potential use in tissue engineering. Eur. Cell Mater. 15: 88-99.

Pittenger MF (2008). Mesenchymal stem cells from adult bone marrow. Methods Mol. Biol. 449: 27-44.

Rahman I, Said DG, Maharajan VS and Dua HS (2009). Amniotic membrane in ophthalmology: indications and limitations. Eye 23: 1954-1961.

Ryan JM, Barry F, Murphy JM and Mahon BP (2007). Interferon-gamma does not break, but promotes the immunosuppressive capacity of adult human mesenchymal stem cells. Clin. Exp. Immunol. 149: 353-363.

Swieszkowski W, Tuan BH, Kurzydlowski KJ and Hutmacher DW (2007). Repair and regeneration of osteochondral defects in the articular joints. Biomol. Eng. 24: 489-495.

Tan SL, Sulaiman S, Pingguan-Murphy B, Selvaratnam L, et al. (2011). Human amnion as a novel cell delivery vehicle for chondrogenic mesenchymal stem cells. Cell Tissue Bank. 12: 59-70.

Wakitani S, Goto T, Pineda SJ, Young RG, et al. (1994). Mesenchymal cell-based repair of large, full-thickness defects of articular cartilage. J. Bone Joint Surg. Am. 76: 579-592.

Wakitani S, Imoto K, Yamamoto T, Saito M, et al. (2002). Human autologous culture expanded bone marrow mesenchymal cell transplantation for repair of cartilage defects in osteoarthritic knees. Osteoarthritis Cartilage 10: 199-206.

Zhang L and Chan C (2010). Isolation and enrichment of rat mesenchymal stem cells (MSCs) and separation of singlecolony derived MSCs. J. Vis. Exp. 22: pii: 1852. Doi: 10.3791/1852. 